\title{
Polygenes and Modifier Genes for Tetracycline and Penicillin Resistance in Neisseria gonorrhoeae
}

\author{
By PATRICIA F. WARNER, LEONARD J. ZUBRZYCKI* \\ AND MARIA CHILA \\ Department of Microbiology and Immunology, Temple University \\ School of Medicine, Philadelphia, Pennsylvania 19140, U.S.A.
}

(Received 11 April 1979; revised 26 August 1979)

\begin{abstract}
The genetic basis for spontaneous resistance to tetracyline (Tet) and penicillin (Pen) in Neisseria gonorrhoeae was investigated. Tet and pen are polygenes which confer small but distinct levels of resistance to Tet and Pen, respectively. Mtr is a multiple-drug resistance polygene which increases resistance to Tet and Pen (as well as to other unrelated antibiotics). Tem is a modifier gene affecting resistance to Tet and Pen. Pem is a modifier gene for Pen resistance.

The following gene combinations code for resistance to five antibiotics: tet, $m t r$ and tem for Tet; pen, $m t r$, pem and tem for Pen; tet, tem and $m t r$ for doxycycline; pen and pem for ampicillin; pen, pem and $m t r$ for nafcillin.
\end{abstract}

\section{INTRODUCTION}

Transformation experiments with Neisseria gonorrhoeae demonstrate that different levels of resistance to penicillin (Pen) and tetracycline (Tet) are due to individual genes and a combination of genes (Maier et al., 1977; Maier et al., 1975a, b; Sarubbi et al., 1974; Sparling et al., 1975). The pen gene or pen $\mathrm{A}$ and another, $m$ tr or ery-2, code for different low levels of resistance to Pen (Sparling, 1977; Sparling et al., 1975). In combination, these genes have additive effects. Another gene, pen $\mathrm{B}$, together with the two Pen resistance genes results in a further increase in resistance to penicillin (Sparling et al., 1975). The combination of these three genes still does not account for the level of resistance to Pen which is exhibited by the multiple-drug-resistant DNA donor strain. With regard to Tet resistance, the tet gene codes for a low level of resistance (Maier et al., 1975a, b; Sparling et al., 1975). This gene together with pen $\mathrm{B}$ and $m t r$ account for the full donor level resistance to Tet (Maier et al., 1975b; Sparling et al., 1975).

We also have detected a gene, tem, which enhances Tet and Pen resistance, but, unlike pen $\mathrm{B}$, it has no phenotypic expression of its own. Tem is by definition a modifier gene (Bryan, 1961). Another modifier gene, pem, in concert with pen, mtr and tem accounts for the full donor level of resistance to Pen. Tet, $m t r$ and pen are polygenes (Bryan, 1961). The tem and pem genes in combination with appropriate polygenes affect resistance to doxycycline, ampicillin and nafcillin.

\section{METHODS}

Media, chemicals and conditions for cultivation of gonococci were as previously described (Maier et al., 1975a).

Bacterial strains. The multiple-drug-resistant strain Neisseria gonorrhoeae 24392, the drug-susceptible strain N. gonorrhoeae 49191 (Maier et al., 1975 b) and their derivatives are listed in Table 1. Both 24392 and 49191 are clinical isolates. 
Strain designation

24392

49191

$4 \mathrm{~B} 10$

$6 \mathrm{~T} 20$

MP81

MT93

TM14\$

TM59:

PM50

PM51

PM54

TP32屯

\section{Table 1. Gonococcal strains}

Antibiotic resistance genotype*

tem str tet cml ery mir pen pem

tem $^{+}$str $^{+}$tet $^{+}$cml $^{+}$ery $^{+}$mtr $^{+}$pen $^{+}$pem $^{+}$

Genotype and phenotype $\left(\mu \mathrm{g} \mathrm{ml}^{-1}\right)$ relevant to Tet and Pen

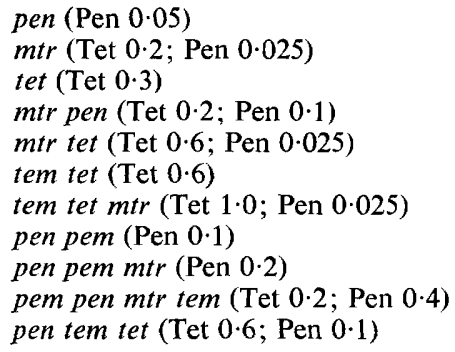

Source

Clinical isolate

Clinical isolate

Transformation $\dagger$

(Donor $\rightarrow$ recipient)

$$
\begin{aligned}
& 24392 \rightarrow 49191 \S \\
& 24392 \rightarrow 49191 \S \\
& 24392 \rightarrow 49191 \S \\
& 4 \mathrm{~B} 10 \rightarrow 7 \mathrm{P} 16 \S \\
& 4 \mathrm{~B} 10 \rightarrow 6 \mathrm{~T} 20 \S \\
& 24392 \rightarrow 49191 \\
& 4 \mathrm{~B} 10 \rightarrow \text { TM14 } \\
& 24392 \rightarrow 49191 \\
& \text { PM50 } \rightarrow \text { 4B10 } \\
& \text { TM14 } \rightarrow \text { PM51 } \\
& 7 \text { P16 } \rightarrow \text { TM14 }
\end{aligned}
$$

* + indicates wild-type or susceptibility to antibiotics. The following genes code for resistance to antibiotics $\left(\mu \mathrm{g} \mathrm{ml}^{-1}\right)$ : str $1000 ;$ tet $0.3 ; \mathrm{cml} 1.0$; er y 0.25 ; pen $0.05 ; \mathrm{mtr}$, a multiple-drug resistance gene for Tet 0.2 , Cml 0.5; Ery 1.0, Pen 0.025; pem, modifier for Pen; tem, modifier for Tet and Pen.

$\dagger$ The genes were added sequentially except in the case of pen pem which are closely linked and were cotransferred.

\$ TM14, TM59 and TP32 also possess the str, $\mathrm{cml}$ and ery (low level) resistance genes of linkage group I which do not affect Tet or Pen resistance (Maier et al., 1975b).

$\S$ Maier et al. $(1975 a)$.

Isogenic crosses. Strain 24392 was the parent for all the genes listed. These genes were individually transformed into strain 49191 . The genetic basis for antibiotic resistance presented is based on isogenic crosses between strains of 49191 .

Extraction of DNA. For standard transformation procedures, DNA was extracted by a phenol method (Young \& Wilson, 1974). For rapid transformation procedures, a crude DNA preparation was used (Wharton \& Zubrzycki, 1976).

Standard transformation. The standard transformation procedure previously described (Maier et al., 1975a) was modified by using phenol-extracted $\mathrm{DNA}$ and $2 \mathrm{~mm}-\mathrm{CaCl}_{2}$ in the transformation mix. These modifications allowed detection of greater linkage between genes (Warner et al., 1975).

Rapid transformation. The rapid transformation procedure (Zubrzycki \& Robinson, 1978) involved spotting crude DNA preparations on to a lawn previously spread with a competent recipient, using either a standard inoculating loop or a 25-pronged replicator. The use of the latter allows the delivery of duplicate preparations of DNA and broth controls on to a variety of antibiotic-containing plates.

Phenotypic lag. For selecting antibiotic-resistant transformants, a 4 to $6 \mathrm{~h}$ lag time was allowed before the agar with micro-colonies was layered on to antibiotic-containing media (Maier et al., 1975a).

The Tet and Pen resistance genotypes. The genes for Tet and Pen resistance were identified by the levels of resistance conferred (Maier et al., 1975a). The genotypes and phenotypes with regard to Tet and Pen resistance are shown in Table 1.

Minimal inhibitory concentrations (m.i.c.). The procedure for determining the m.i.c. values of antibiotics involved a multipronged replicator system which allows delivery of many cell suspensions on to plates containing different concentrations of antibiotics (Maier et al., 1975 a). To ensure reproducibility and proper evaluation of results, the following strains with known antibiograms were used: 24392, 49191, 7P16, 4B10, 6T20, MP81, MT93. M.i.c. values for Tet were determined by testing at concentrations of $\left(\mu \mathrm{g} \mathrm{ml}^{-1}\right): 0 \cdot 15$, $0 \cdot 2,0 \cdot 25,0 \cdot 3,0 \cdot 5,1 \cdot 0$ and $1 \cdot 5$. Those for Pen were: $0 \cdot 01,0 \cdot 015,0 \cdot 02,0 \cdot 025,0 \cdot 03,0 \cdot 05,0 \cdot 1,0 \cdot 12,0 \cdot 15,0 \cdot 2$, 0.4 and $0 \cdot 8$.

\section{RES ULTS}

\section{Tem, a modifier locus affecting tetracycline resistance}

Previous work using sodium dodecyl sulphate (SDS)-prepared DNA established the genotypes and phenotypes of three classes of Tet-resistant transformants (Maier et al., 


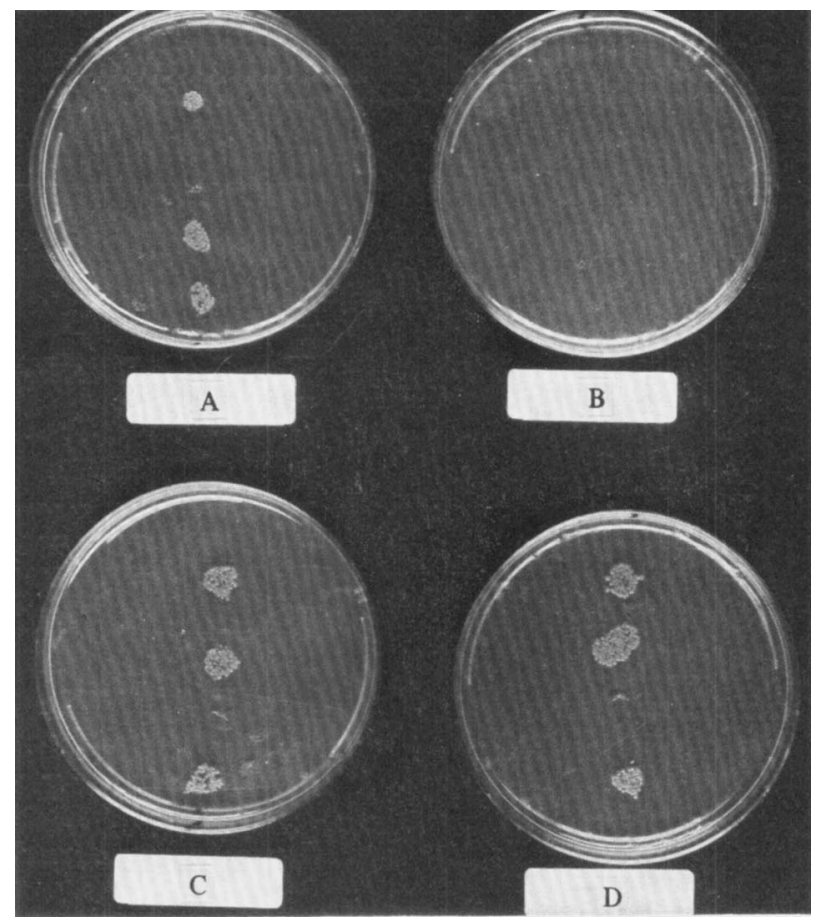

Fig. 1. Results of an experiment in which DNA from four strains was spotted with a loop on to agar plates previously spread with a suspension of gonococci and then layered, after a $5 \mathrm{~h}$ phenotypic lag, on to agar containing different concentrations of Tet $\left(\mu \mathrm{g} \mathrm{ml}^{-1}\right)$ as follows: plate A, 49191, $0 \cdot 3$; plate B, 49191, 0.6; plate C, $6 \mathrm{~T} 20,0 \cdot 6$; plate D, TM14, $1 \cdot 0$. On plates A, B and C the DNA spotted (from top to bottom) was extracted from strains TM14, 4B10, MT93 and 24392. On plate D the DNA came from MT93, 4B10, 6T20 and 24392.

1975b). Typical strains (4B10, 6T20 and MT93) are listed in Table 1. Using phenol-extracted DNA, identical strains were isolated, but in addition, a fourth class, represented by TM14 (Table 1), was observed.

Transformants MT93 and TM14 (Table 1) exhibited the same level of resistance to Tet, but differed in the presence or absence of the $m t r$ gene (characterized by resistance to erythromycin at $1 \mu \mathrm{g} \mathrm{ml}^{-1}$ ). It was deduced that a third locus, called $t e m$, was involved in Tet resistance.

In order to establish the role of tem in Tet resistance, rapid transformation experiments were conducted. The results from part of one such experiment are shown in Fig. 1. Plate A shows that the $0 \cdot 3 \mu \mathrm{g} \mathrm{ml}^{-1}$ level of resistance is conferred by tet $\left(0 \cdot 3 \mu \mathrm{g} \mathrm{ml}^{-1}\right)$ from TM 14 or MT93 but not $m \operatorname{tr}\left(0 \cdot 2 \mu \mathrm{g} \mathrm{ml}^{-1}\right)$ from 4B10. The absence of growth spots on plate B shows that the $0.6 \mu \mathrm{g} \mathrm{ml}^{-1}$ level of resistance requires two or more genes which cannot be transferred simultaneously into 49191 by this technique. Plate $C$ shows that the $0.6 \mu \mathrm{g} \mathrm{ml}^{-1}$ level of resistance can be attained if the recipient $6 \mathrm{~T} 20$, which contains tet $\left(0.3 \mu \mathrm{g} \mathrm{ml}^{-1}\right)$, receives tem (no phenotype) from TMl4 or $m t r\left(0 \cdot 2 \mu \mathrm{g} \mathrm{ml}^{-1}\right)$ from 4B10. The 6T20 DNA is a negative control. Plate $\mathrm{D}$ shows that an even higher level of resistance $\left(1 \mu \mathrm{g} \mathrm{ml}^{-1}\right)$ can be attained if the recipient TM14, which contains tet $\left(0.3 \mu \mathrm{g} \mathrm{ml}^{-1}\right)$ and tem (no phenotype), receives $m t r\left(0.2 \mu \mathrm{g} \mathrm{ml}^{-1}\right)$ from MT93 or $4 \mathrm{~B} 10$. The tet-tem combination (6T20-TM14) does not confer this level of resistance. DNA from 24392 containing all the Tet genes was used as a control. Analyses of these results is an example of how the existence of a tem locus can be detected by using strains with known genotypes as recipient and DNA donors in a variety of genetic crosses. The results of a complete experiment using a multipronged replicator are shown in Table 2. The use of 24392 DNA, containing the tet, mtr and tem 
Table 2. Association of different genotypes with the various phenotypic classes of tetracycline-resistant strains obtained from transformation experiments

Bacterial lawn*

[gene(s) and level of resistance to Tet $\left.\left(\mu \mathrm{g} \mathrm{ml}^{-1}\right)\right]$

$6 \mathrm{~T} 20($ tet $0 \cdot 3)$

$4 \mathrm{~B} 10(m t r 0 \cdot 2)$

MT93 (mtr tet 0.6$)$

TM14 (tet tem $0 \cdot 6$ )

49191 (sensitive wild-type $0 \cdot 1$ ) $\begin{array}{ccc}\begin{array}{c}\text { Selection } \\ \text { plate } \\ \text { with Tet } \\ \left(\mu \mathrm{g} \mathrm{ml}^{-1}\right)\end{array} & \text { 6T20 4B10 MT93 TM14 } 2439249191\end{array}$

$\begin{array}{lllllll}0.3 & + & + & + & + & + & + \\ 0.6 & - & + & + & + & + & - \\ 0.8 & - & - & - & - & - & - \\ 0.3 & + & - & + & + & + & - \\ 0.6 & + & - & + & + & + & - \\ 0.8 & - & - & - & - & - & - \\ 0.8 & - & - & - & + & + & - \\ 1.0 & - & - & - & + & + & - \\ 1.5 & - & - & - & - & - & - \\ 0.8 & - & + & + & - & + & - \\ 1.0 & - & + & + & - & + & - \\ 1.5 & - & - & - & - & - & - \\ 0.2 & + & + & + & + & + & - \\ 0.3 & + & - & + & + & + & - \\ 0.6 & - & - & - & - & - & -\end{array}$

* A lawn of 24392 grew on all Tet plates except $1.5 \mu \mathrm{g} \mathrm{ml}^{-1}$.

$\dagger+$, Growth; -, no growth. Growth means that the transformants were resistant to Tet at the concentration in the plate, as indicated.

genes, shows that no more than one Tet resistance gene can beltransformed by this rapid technique. DNA from 49191 is the negative control. In all cases DNA from one strain spotted on a lawn of that same strain did not increase its level of resistance to Tet. This means that a 'double dose effect' does not occur. The tet in 6T20, MT93 and TM14 DNA preparations has additive effects with the $m t r$ in $4 \mathrm{~B} 10$, increasing its level of resistance from 0.3 to $0.6 \mu \mathrm{g} \mathrm{ml}^{-1}$. The same effect occurs using $m$ tr DNA (4B10 or MT93) in combination with tet (6T20). The tet +tem in TM14 DNA has additive effects with the tet in 6T20 and with the $m t r+t e t$ combination in MT93, increasing the Tet resistance of these strains to the levels shown in Table 2. 'Double dose effects' do not occur; therefore, these results are due to the $t e t+t e m$ and $t e t+m t r+t e m$ combinations, respectively. The latter genotype with the same phenotypic result can be constructed by adding $m t r$ from 4B10 to tet $+t e m$ in TM14. Such a strain, TM59 (Table 1), exhibits the same level of resistance to Tet as does strain 24392. It should be reported that results similar to those in Table 2 were obtained with many other isolates (Warner, 1978).

Although the effects of tem on Tet resistance are obvious, the tem gene has not been isolated alone in any strain. Many attempts to do so after rapid or standard transformation experiments have been unsuccessful (Warner, 1978). The most likely explanation is that tem has no phenotypic expression of its own.

The results obtained and conclusions reached as a result of the rapid transformation experiments were confirmed by standard transformation experiments. In fact, the strains used to obtain the results shown in Table 2 were also obtained from standard transformation experiments.

\section{Linkage of the tet and tem loci}

The nature of the rapid spotting technique using a crude DNA preparation and small amounts of DNA per spot does not permit the transfer of more than one Tet resistance gene at a time. However, in standard transformation experiments using phenol-extracted DNA from TM14, transformants resistant to Tet at the $0.6 \mu \mathrm{g} \mathrm{ml}^{-1}$ level occurred at a frequency $\left(10^{-3}\right)$ associated with a single transformation event (Maier et al., 1975a). This 
Table 3. Association of different genotypes with the various phenotypic classes of penicillin-resistant strains obtained from transformation experiments

\begin{tabular}{|c|c|c|c|c|c|}
\hline \multirow{4}{*}{$\begin{array}{l}\text { Bacterial lawn } \\
\text { [gene(s) and level of } \\
\text { resistance to } \\
\left.\text { Pen }\left(\mu \mathrm{g} \mathrm{ml}^{-1}\right)\right]\end{array}$} & \multirow{4}{*}{$\begin{array}{l}\text { Selection } \\
\text { plate } \\
\text { with Pen } \\
\left(\mu \mathrm{g} \mathrm{ml}^{-1}\right)\end{array}$} & \multicolumn{4}{|c|}{ Growth in area in which DNA was spotted } \\
\hline & & \multirow{3}{*}{$\begin{array}{l}4 \mathrm{~B} 10 \\
(m t r)\end{array}$} & \multicolumn{2}{|c|}{ Source of DNA } & \multirow{3}{*}{$\begin{array}{c}\text { MP81 } \\
(\text { mtr pen) }\end{array}$} \\
\hline & & & $7 \mathrm{P} 16$ & PM50 & \\
\hline & & & (pen) & (pen pem) & \\
\hline \multirow[t]{3}{*}{ Type A (pen 0.05) } & $0 \cdot 1$ & + & - & + & + \\
\hline & $0 \cdot 2$ & - & - & - & - \\
\hline & 0.4 & - & - & - & - \\
\hline \multirow[t]{3}{*}{ Type B $(p e n+p e m ~ 0 \cdot 1)$} & $0 \cdot 1$ & + & - & + & + \\
\hline & $0 \cdot 2$ & + & - & - & + \\
\hline & $0 . \overline{4}$ & - & - & - & - \\
\hline
\end{tabular}

level of resistance in these strains is due to two genes, tet and tem. Their presence in these transformants was confirmed by rapid transformation tests. The close linkage of the tet and tem loci was confirmed by routine DNA dilution experiments (Goodgal, 1961). DNA from strain TM14 was used to transform strain 49191. Below the saturating concentration of $1.0 \mu \mathrm{g} \mathrm{DNA} \mathrm{ml}^{-1}$, the number of Tet-resistant transformants at the $0.6 \mu \mathrm{g} \mathrm{ml}^{-1}$ level decreased in linear proportion to the decreasing DNA concentration (Warner, 1978). Therefore, these Tet-resistant transformants have received only one piece of DNA which carries the two loci tet and tem.

\section{Pem, a modifier locus affecting penicillin resistance}

As a result of transformation experiments using SDS-extracted DNA, three phenotypic classes of Pen-resistant transformants were observed by Maier et al. (1975b). The representative strains 4B10, 7P16 and MP81 are listed in Table 1. Identical transformants were also obtained when phenol-extracted DNA was used. In addition, other classes of transformants were obtained. The class of transformants represented by strain PM50 was of considerable interest. With regard to Pen resistance, MP81 and PM50 differ only in the presence or absence of $m t r$ (characterized by resistance to erythromycin at $1 \mu \mathrm{g} \mathrm{ml}^{-1}$ ). It was deduced that another locus, labelled pem, was involved in Pen resistance.

From standard transformation experiments using PM50 DNA, only two phenotypic classes of Pen-resistant transformants were isolated. Type A was resistant to $0.05 \mu \mathrm{g} \mathrm{ml}^{-1}$ and presumed to contain pen, and type B was resistant to $0.1 \mu \mathrm{g} \mathrm{ml}^{-1}$ and presumed to contain pen and pem. These two types were used in rapid transformation experiments. Because $m t r$ alone confers a low level of Pen resistance, the effect of this gene on the type A and type B transformants was also determined. The results (Table 3 ) indicate that a third locus, pem, does account for an increase in the level of resistance to Pen. Attempts to isolate pem in strains obtained from rapid and standard transformation experiments have been unsuccessful (Warner, 1978). Similar to tem, the pem locus has no phenotypic expression of its own other than enhancing the resistance expressed by pen and $m t r$.

The results of these rapid and subsequently standard transformation experiments prove that the combination of pen, pem and $m t r$ codes for resistance to Pen at $0.2 \mu \mathrm{g} \mathrm{ml}^{-1}$. However, this gene combination does not produce the full level of resistance to Pen of $0.4 \mu \mathrm{g} \mathrm{ml}^{-1}$ observed in strain 24392. It was deduced that at least one other gene was involved in Pen resistance.

\section{The tem locus affects penicillin resistance}

Rapid transformation experiments showed that DNA from TM14 in combination with pen, pem and $m t r$ in PM51 resulted in transformants, such as PM54 (Table 1), which exhibit the full level of resistance to Pen as observed for strain 24392. TM14 contains tem 
Table 4. Role of the tem and pem loci in cross-resistance of 49191 transformants to other antibiotics*

\begin{tabular}{|c|c|c|c|}
\hline Strain & Ampicillin & Nafcillin & Doxycycline \\
\hline 49191 & 0.06 & 0.6 & $0 \cdot 25$ \\
\hline 24392 & 0.25 & 15 & $2 \cdot 0$ \\
\hline $6 \mathrm{~T} 20(\mathrm{tet})$ & 0.06 & 0.6 & $1 \cdot 0$ \\
\hline $4 \mathrm{~B} 10(\mathrm{mtr})$ & 0.06 & $1 \cdot 25$ & 0.5 \\
\hline $7 \mathrm{P} 16$ (pen) & $0 \cdot 15$ & $2 \cdot 5$ & $0 \cdot 25$ \\
\hline MP81 (mtr pen) & $0 \cdot 15$ & 10 & 0.5 \\
\hline MT93 (mtr tet) & 0.06 & $1 \cdot 25$ & $1 \cdot 5$ \\
\hline PM50 (pem pen) & 0.25 & 10 & 0.25 \\
\hline PM51 (mtr pem pen) & 0.25 & 15 & $0 \cdot 5$ \\
\hline TM14 (tet tem) & 0.06 & 0.6 & $1 \cdot 0$ \\
\hline TM59 (mtr tet tem) & 0.06 & $1 \cdot 25$ & $2 \cdot 0$ \\
\hline
\end{tabular}

and tet, but tet does not confer resistance to Pen (Maier ${ }^{-}$et al., 1975a, b; Warner, 1978). Since only one gene can be transferred, it had to be tem which increased resistance to Pen. These results were confirmed by standard transformation experiments.

\section{Linkage of the pen and pem loci}

DNA from PM50 (pen pem) was used to transform Pen resistance genes into 49191. The pen and pem markers cotransfer $90 \%$ of the time at a frequency of $10^{-3}$ which is associated with a single transformational event (Maier et al., 1975a). A DNA dilution curve also confirmed that transformants resistant to Pen at $0 \cdot 1 \mu \mathrm{g} \mathrm{ml}^{-1}$ received only one piece of DNA, which carries the two genes pen and pem (Warner, 1978). Therefore, the pem locus is linked to pen.

\section{Cross-resistance of transformants to doxycycline, ampicillin and nafcillin}

Transformants which carry a single antibiotic resistance locus or a specific two-gene combination exhibit cross-resistance to other antibiotics and antibacterial agents (Maier et al., 1975b). However, the gene combinations previously reported did not account for the full level of resistance of 24392 to a semi-synthetic tetracycline (doxycycline), a broad spectrum penicillin (ampicillin) and a $\beta$-lactamase-resistant penicillin (nafcillin). M.i.c. analyses of many transformants demonstrated that the tem and pem loci were necessary for the full level of resistance to these antibiotics. As shown in Table 4, the following gene combinations code for cross-resistance to the three antibiotics: doxycycline, tet tem $\mathrm{mtr}$; ampicillin, pen pem; nafcillin, pen pem mtr. In addition, the current studies confirm the phenotypic effects of the individual genes tet, $m$ tr and pen for these three antibiotics (Maier et al., 1975b).

\section{DISCUSSION}

Genetic analyses of two clinical isolates and a laboratory-induced mutant of $N$. gonorrhoeae has led to the identification of chromosomal loci responsible for resistance to a variety of antibacterial agents (Maier et al., 1975b; Sparling, 1977). However, the genetic basis for resistance to Tet, Pen and related antibiotics was not completely established.

It was reported that transformants with a variety of phenotypic patterns were observed in mixed-culture experiments (Warner et al., 1975). These phenotypes were not observed from standard transformation experiments previously reported (Maier et al., 1975a,b). The results of mixed-culture experiments suggested the possible genotypes which could 
account for the donor (24392) level of resistance to Tet and Pen. However, it was difficult to determine the genes involved because of the plethora of genetic exchanges which occur in mixed cultures. The genetic basis for resistance could only be established from controlled experimental situations.

An essential approach for resolving the interplay of genotypes controlling Tet and Pen resistance was the use of strains containing individual genes or combinations of genes coding for distinct levels of resistance to antibiotics. For example, the phenotypic effects of the tet $+t e m$ combination could not be distinguished from that of $t e t+m t r$ without using strains known to contain only tet or mtr (see Table 1). The selection and use of strains containing only $m t r$ or pen allowed us to establish three different genotypes which result in the same phenotype (see Table 1).

An important technical approach was the use of the rapid transformation procedure. This allowed us to test the effects of various combinations of genotypes before confirming the results by standard transformation experiments. With the rapid transformation and m.i.c. procedures about 2500 transformants were tested during the various stages of the experiments described. Therefore, the conclusions presented are not based merely on the results obtained with a few strains. Most of the work on the genetic basis for antibiotic resistance of $N$. gonorrhoeae conducted in our laboratory and that of Dr P. F. Sparling are in agreement (Maier et al., 1977; Maier et al., 1975b; Sparling, 1977). Even the pen B and tem loci are functionally similar. However, there are important differences.

From studies involving pen $\mathrm{B}$, it was concluded that ery-2, now designated $m$ tr (Sparling, 1977), is a modifier gene for pen B. This gene combination results in an increased resistance to Tet and Pen. However, all recipients in these studies of the pen $\mathrm{B}$ gene contained $m t r$ $($ ery-2) and other antibiotic resistance genes. Therefore, it is not clear whether mtr (ery-2) or pen $\mathrm{B}$ is a modifier. In these studies it was found that $m$ tr $($ ery-2) confers a low level of resistance to Pen and a slight, almost indistinguishable, level of resistance to Tet. It may be that the concentrations of Tet used in the m.i.c. test did not allow the investigators to associate a distinct level of Tet resistance with $m t r$ (ery-2). In our current and previous studies (Maier et al., 1975b) $\mathrm{mtr}$ has been shown to confer a distinct level of resistance to Tet and Pen. As concluded herein, the tem gene is a modifier gene (Bryan, 1961) enhancing resistance to Tet and Pen conferred by $m t r$, tet or pen, but having no other detectable phenotypic expression of its own. With regard to Tet and Pen, tet, mtr and pen are polygenes which make a small but distinct contribution to the phenotype of an organism (Bryan, 1961).

It may be that tem and pen $\mathrm{B}$ are different genes. By three-factor crosses pen $\mathrm{B}$ was found to be linked to the $s p c$ (spectinomycin) and $\mathrm{cml}$ (chloramphenicol) loci (Sparling et al., 1975). The fact that tem is cotransferred with tet suggests that it is distal to the $s p c-c m l$ region. Only a comparative study of the pen $\mathrm{B}$ and tem genes in a common recipient will determine whether they are different genes with functional similarities.

No gene similar to, nor a functional equivalent of, the pem gene has been reported. Like the tem gene, it is a modifier for gonococcus. These two modifier genes in combination with $m t r$ and pen account for the full level of donor resistance to Pen. It should be noted that neither strain 24392 nor 49191 produces $\beta$-lactamase (Warner, 1978) as determined by an iodometric test (Catlin, 1975). This, plus the fact that 24392 and all transformants tested are stably resistant to Pen, Tet and the other antibiotics referred to, is consistent with the proposition that these genes are chromosomally located. However, one cannot eliminate the possibility that antibiotic resistance genes of $N$. gonorrhoeae are plasmid determined (Sparling, 1977).

Reyn (1961) reported that gonococci which are resistant to one antibacterial agent tend to be resistant to others. The genetic basis for this cross-resistance can be ascertained from previous publications (Maier et al., 1975b; Sparling, 1977). The modifier genes tem and pem can be added to the list of genes which in combination with other genes account for cross-resistance to doxycycline, ampicillin and nafcillin. 
This research was supported by a faculty grant-in-aid for research from Temple University, and by RR05417, an N.I.H. grant.

\section{REFERENCES}

BRYAN, B. E. (1961). Genetic modifiers of streptomycin resistance in pneumococcus. Journal of Bacteriology 82, 461-470.

Catlin, B. W. (1975). Iodometric detection of Haemophilus influenzae beta-lactamase: rapid presumptive test for ampicillin resistance. Antimicrobial Agents and Chemotherapy 7, 265-270.

GoodgaL, S. H. (1961). Studies on transformation of Haemophilus influenzae. IV. Linked and unlinked transformation. Journal of General Physiology 45, 205-228.

MaIER, T. W., Zubrzycki, L. \& Coyle, M. B. $(1975 a)$. Genetic analysis of drug resistance in Neisseria gonorrhoeae: identification and linkage relationships of loci controlling drug resistance. Antimicrobial Agents and Chemotherapy 7, 676681.

Maier, T. W., Zubrzycki, L., Coyle, M. B., Chila, M. \& WARNER, P. $(1975 b)$. Genetic analysis of drug resistance in Neisseria gonorrhoeae: production of increased resistance by the combination of two antibiotic resistance loci. Journal of Bacterio$\log y$ 124, 834-842.

Maier, T. W., Warner, P., Zubrzycki, L. \& Chila, M. (1977). Identification of drug resistance loci in various clinical isolates of Neisseria gonorrhoeae. Antimicrobial Agents and Chemotherapy 12, 444. 446.

ReYN, A. (1961). Sensitivity of $N$. gonorrhoeae to antibiotics. British Journal of Venereal Diseases 37, 145-157.

Sarubbi, F. A., Blackman, E. \& Sparling, P. F. (1974). Genetic mapping of linked antibiotic resistance loci in Neisseria gonorrhoeae. Journal of Bacteriology 120, 1284-1292.
Sparling, P. F. (1977). Antibiotic resistance in the gonococcus. In The Gonococcus, pp. 112-135. Edited by R. B. Roberts. New York: John Wiley.

Sparling, P. F., Sarubbi, F. A. \& Blackman, E. (1975). Inheritance of low level resistance to penicillin, tetracycline and chloramphenicol in Neisseria gonorrhoeae. Journal of Bacteriology 124, 740-749.

WARNER, P. F. (1978). Genetic analysis of multiple drug resistance of Neisseria gonorrhoeae: polygenic higher-level tetracycline and penicillin resistance. Ph.D. thesis, Temple University, Philadelphia, U.S.A.

WAR Ner, P., Zubrzycki, L., MAIER, T. \& ChILA, M. (1975). Antibiotic resistance genes of Neisseria gonorrhoeae. In Abstracts of the Annual Meetings of the American Society for Microbiology, p. 97. Edited by H. Gooder. Washington, D.C.: American Society for Microbiology.

Wharton, R. D. \& ZubrzyCKI, L. (1976). Temperature sensitive mutants of Neisseria gonorrhoeae. Journal of Bacteriology 127, 1579-1581.

Young, F. E. \& Wilson, G. A. (1974). Genetic analysis of Bacillus subtilis. In Bacteria, Bacteriophages and Fungi, Handbook of Genetics, pp. 69114. Edited by R. C. King. New York: Plenum Press.

ZUBRYZCKI, L. \& RoBINSON, D. (1978). Use of conditional lethal mutants in genetic studies of Neisseria gonorrhoeae. In Immunobiology of Neisseria gonorrhoeae, pp. 53-54. Edited by G. F. Brooks, E. C. Gotschlich, K. K. Holmes, W. D. Sawyer \& F. E. Young. Washington, D.C.: American Society for Microbiology. 